\title{
Wave dispersion characteristics in lipid tubules considering shell model based on nonlocal strain gradient theory
}

\author{
Chen Liang ${ }^{1}$, Yanqing Wang ${ }^{1}$, and Dongyu $\mathrm{Cao}^{1}$ \\ ${ }^{1}$ Northeastern University College of Sciences
}

August 14, 2020

\begin{abstract}
In this paper, wave dispersion analysis of lipid tubules is presented by using the first-order shear deformation (FSD) shell theory. The small-scale effect is revealed explicitly based on the nonlocal strain gradient theory (NSGT). Different types of lipid tubules with size-dependent material properties are taken into account. Hamilton's principle is utilized to derive the equations of wave motion. The analytical solutions of phase velocity and wave frequency of propagated waves are obtained. In addition, detailed investigations are implemented to highlight the effects of the types of lipid tubules, the longitudinal and circumferential wave numbers, the material length scale parameters and the nonlocal parameters on the wave dispersion characteristics of lipid tubules.
\end{abstract}

\section{Hosted file}

8-\begin\{CJK\}\{UTF8\}\{gbsn\}-\end\{CJK\}\selectlanguage\{english\}5.docx available at https: } //authorea.com/users/350899/articles/475606-wave-dispersion-characteristics-in-lipidtubules-considering-shell-model-based-on-nonlocal-strain-gradient-theory

\section{Hosted file}

Figure. 1.docx available at https://authorea.com/users/350899/articles/475606-wavedispersion-characteristics-in-lipid-tubules-considering-shell-model-based-on-nonlocalstrain-gradient-theory

\section{Hosted file}

Figure. 2.docx available at https://authorea.com/users/350899/articles/475606-wavedispersion-characteristics-in-lipid-tubules-considering-shell-model-based-on-nonlocalstrain-gradient-theory

\section{Hosted file}

Figure. 3.docx available at https://authorea.com/users/350899/articles/475606-wavedispersion-characteristics-in-lipid-tubules-considering-shell-model-based-on-nonlocalstrain-gradient-theory

\section{Hosted file}

Figure. 4.docx available at https://authorea.com/users/350899/articles/475606-wavedispersion-characteristics-in-lipid-tubules-considering-shell-model-based-on-nonlocalstrain-gradient-theory

\section{Hosted file}


Figure. 5.docx available at https://authorea.com/users/350899/articles/475606-wavedispersion-characteristics-in-lipid-tubules-considering-shell-model-based-on-nonlocalstrain-gradient-theory

\section{Hosted file}

Figure. 6.docx available at https://authorea.com/users/350899/articles/475606-wavedispersion-characteristics-in-lipid-tubules-considering-shell-model-based-on-nonlocalstrain-gradient-theory

\section{Hosted file}

Figure. 7.docx available at https://authorea.com/users/350899/articles/475606-wavedispersion-characteristics-in-lipid-tubules-considering-shell-model-based-on-nonlocalstrain-gradient-theory

\section{Hosted file}

Figure. 8.docx available at https://authorea.com/users/350899/articles/475606-wavedispersion-characteristics-in-lipid-tubules-considering-shell-model-based-on-nonlocalstrain-gradient-theory 